Int. J. Open Problems Compt. Math., Vol. 6, No. 4, December 2013 ISSN 1998-6262; Copyright (CICSRS Publication, 2013

www.i-csrs.org

\title{
New Results For a Boundary Value Problem For Caputo Fractional Differential Equations
}

\author{
${ }^{1}$ Louiza TABHARIT and ${ }^{2}$ Zoubir DAHMANI \\ ${ }^{1}$ Department of Math-Info, Faculty SEI, UMAB, Mostaganem \\ e-mail: lz.tabharit@yahoo.fr \\ ${ }^{2}$ Laboratory of Pure and Applied Mathematics, LPAM, \\ Faculty SEI, UMAB of Mostaganem \\ e-mail: zzdahmani@yahoo.fr
}

\begin{abstract}
In this paper, we study the existence and uniqueness of solutions for boundary value problems of fractional differential equations using Caputo approach. The obtained results are proved using the Banach contraction principle and Scheafer fixed point theorem. We also provide some examples to illustrate the possible application of the established results.
\end{abstract}

Keywords: Boundary value problem, Caputo derivative, fixed point theorem. 2010 Mathematics Subject Classification: 34A08, 34A12, 34B25.

\section{Introduction}

Fractional calculus has attracted much interest in the last few decades. This theory has many applications in control theory, edge detection, electrochemistry, electromagnetic, visco-elasticity, dynamics, rheology, and fluid mechanics $[7,10,11,12,19,20]$. The interested reader is referred to the references of Podlubny [15], Kilbas [6], Samko and al [18] for further information and applications. Other recent works on this theory can be founded in $[14,13,18,17]$. Moreover, the investigation for the existence solutions of fractional differential equations have been developed very quickly, see for instance $[1,3,4,5]$.

In this paper we are concerned with the existence and uniqueness of solutions 
for the following problem:

$$
\begin{aligned}
& D^{\alpha} x(t)=f(t, x(t)), \quad t \in[0,1], \\
& a_{1} x(0)+b_{1} x(1)=c_{1}, \\
& a_{2} D^{\beta} x(0)+b_{2} D^{\beta} x(1)=c_{2}, \\
& a_{3} D^{\gamma} x(0)+b_{3} D^{\gamma} x(1)=c_{3},
\end{aligned}
$$

where $D^{\alpha}, D^{\beta}, D^{\gamma}$ denote the Caputo's fractional derivatives with $2<\alpha<3$, $0<\beta<1,1<\gamma<2$, the real constants $a_{1}, a_{2}, a_{3}, b_{1}, b_{2}, b_{3}, c_{1}, c_{2}, c_{3}$ satisfy $a_{1}+b_{1} \neq 0, b_{2} \neq 0, b_{3} \neq 0$ and $f$ is a function that will be specified later.

\section{Preliminaries}

In this section, we give the necessary notation and basic definitions which will be used in this paper.

Definition 2.1: The Riemann-Liouville fractional integral operator of order $\alpha \geq 0$, for a continuous function $f$ on $[0, \infty[$ is defined as

$$
\begin{gathered}
J^{\alpha} f(t)=\frac{1}{\Gamma(\alpha)} \int_{0}^{t}(t-\tau)^{\alpha-1} f(\tau) d \tau ; \quad \alpha>0, t>0, \\
J^{0} f(t)=f(t),
\end{gathered}
$$

where $\Gamma(\alpha):=\int_{0}^{\infty} e^{-u} u^{\alpha-1} d u$.

Definition 2.2: The fractional derivative of $f \in C^{n}([0, \infty[)$ in the Caputo's sense is defined as

$$
D^{\alpha} f(t)= \begin{cases}\frac{1}{\Gamma(n-\alpha)} \int_{0}^{t}(t-\tau)^{n-\alpha-1} f^{(n)}(\tau) d \tau, & n-1<\alpha<n, n \in N^{*} \\ \frac{d^{n}}{d t^{n}} f(t), & \alpha=n .\end{cases}
$$

Details on Caputo's derivative can be found in [15].

Let us now introduce $C(J, \mathbb{R})$ the Banach space of all continuous functions from $J$ into $\mathbb{R}$ with the norm $\|x\|=\sup _{t \in J}|x(t)|$.

We give the following two lemmas [18]:

Lemma 2.1 For $\alpha>0$, the general solution of the fractional differential $D^{\alpha} x=0$ is given by

$$
x(t)=c_{0}+c_{1} t+c_{2} t^{2}+\ldots c_{n-1} t^{n-1},
$$

where $c_{i} \in \mathbb{R}, i=0,1,2, \ldots n-1, n=[\alpha]+1$.

Lemma 2.2 Let $\alpha>0$, then

$$
J^{\alpha} D^{\alpha} x(t)=x(t)+c_{0}+c_{1} t+c_{2} t^{2}+\ldots c_{n-1} t^{n-1},
$$

for some $c_{i} \in \mathbb{R}, i=0,1,2, \ldots n-1, n=[\alpha]+1$. 
We prove the following result:

Lemma 2.3 Let $2<\alpha<3$. A solution of the problem (1) is given by:

$$
\begin{gathered}
x(t)=J^{\alpha} f(t, x(t))-\frac{b_{1}}{a_{1}+b_{1}}\left\{\int_{0}^{1} \frac{(1-\tau)^{\alpha-1}}{\Gamma(\alpha)} f(\tau, x(\tau)) d \tau\right. \\
-\Gamma(2-\beta)\left[\int_{0}^{1} \frac{(1-\tau)^{\alpha-\beta-1}}{\Gamma(\alpha-\beta)} f(\tau, x(\tau)) d \tau-\frac{c_{2}}{b_{2}}\right] \\
\left.-\frac{\beta}{2-\beta}\left[\int_{0}^{1} \frac{(1-\tau)^{\alpha-\gamma-1}}{\Gamma(\alpha-\gamma)} f(\tau, x(\tau)) d \tau-\frac{c_{3}}{b_{3}} \Gamma(3-\gamma)\right]\right\} \\
-\frac{c_{1}}{a_{1}+b_{1}}-\left\{\Gamma(2-\beta)\left[\int_{0}^{1} \frac{(1-\tau)^{\alpha-\beta-1}}{\Gamma(\alpha-\beta)} f(\tau, x(\tau)) d \tau-\frac{c_{2}}{b_{2}}\right]\right. \\
\left.-\frac{\Gamma(3-\gamma)}{(2-\beta)}\left[\int_{0}^{1} \frac{(1-\tau)^{\alpha-\gamma-1}}{\Gamma(\alpha-\gamma)} f(\tau, x(\tau)) d \tau-\frac{c_{3}}{b_{3}}\right]\right\} t \\
-\left\{\frac{\Gamma(3-\gamma)}{2}\left[\int_{0}^{1} \frac{(1-\tau)^{\alpha-\gamma-1}}{\Gamma(\alpha-\gamma)} f(\tau, x(\tau)) d \tau-\frac{c_{3}}{b_{3}}\right]\right\} t^{2} .
\end{gathered}
$$

Proof We use lemmas 2.1 and 2.2 for $2<\alpha<3$. We can write:

$$
x(t)=J^{\alpha} f(t, x(t))-k_{0}-k_{1} t-k_{2} t^{2} .
$$

By the conditions of (1), we obtain

$$
\begin{aligned}
k_{0}= & \frac{b_{1}}{a_{1}+b_{1}}\left\{\int_{0}^{1} \frac{(1-\tau)^{\alpha-1}}{\Gamma(\alpha)} f(\tau, x(\tau)) d \tau\right. \\
& -\Gamma(2-\beta)\left[\int_{0}^{1} \frac{(1-\tau)^{\alpha-\beta-1}}{\Gamma(\alpha-\beta)} f(\tau, x(\tau)) d \tau-\frac{c_{2}}{b_{2}}\right] \\
& \left.-\frac{\beta}{2-\beta}\left[\int_{0}^{1} \frac{(1-\tau)^{\alpha-\gamma-1}}{\Gamma(\alpha-\gamma)} f(\tau, x(\tau)) d \tau-\frac{c_{3}}{b_{3}} \Gamma(3-\gamma)\right]\right\}-\frac{c_{1}}{a_{1}+b_{1}},
\end{aligned}
$$




$$
\begin{aligned}
k_{1}= & \Gamma(2-\beta)\left[\int_{0}^{1} \frac{(1-\tau)^{\alpha-\beta-1}}{\Gamma(\alpha-\beta)} f(\tau, x(\tau)) d \tau-\frac{c_{2}}{b_{2}}\right] \\
& -\frac{\Gamma(3-\gamma)}{(2-\beta)}\left[\int_{0}^{1} \frac{(1-\tau)^{\alpha-\gamma-1}}{\Gamma(\alpha-\gamma)} f(\tau, x(\tau)) d \tau-\frac{c_{3}}{b_{3}}\right], \\
k_{2}= & \frac{\Gamma(3-\gamma)}{2}\left[\int_{0}^{1} \frac{(1-\tau)^{\alpha-\gamma-1}}{\Gamma(\alpha-\gamma)} f(\tau, x(\tau)) d \tau-\frac{c_{3}}{b_{3}}\right] .
\end{aligned}
$$

Lemma 2.3 is thus proved.

To prove our results, we need to define the operator $\Psi: C([0,1], \mathbb{R}) \longrightarrow$ $C([0,1], \mathbb{R})$ as follows:

$$
\begin{gathered}
\Psi x(t)=J^{\alpha} f(t, x)-\frac{b_{1}}{a_{1}+b_{1}}\left\{\int_{0}^{1} \frac{(1-\tau)^{\alpha-1}}{\Gamma(\alpha)} f(\tau, x(\tau)) d \tau\right. \\
-\Gamma(2-\beta)\left[\int_{0}^{1} \frac{(1-\tau)^{\alpha-\beta-1}}{\Gamma(\alpha-\beta)} f(\tau, x(\tau)) d \tau-\frac{c_{2}}{b_{2}}\right] \\
\left.-\frac{\beta}{2-\beta}\left[\int_{0}^{1} \frac{(1-\tau)^{\alpha-\gamma-1}}{\Gamma(\alpha-\gamma)} f(\tau, x(\tau)) d \tau-\frac{c_{3}}{b_{3}} \Gamma(3-\gamma)\right]\right\} \\
-\frac{c_{1}}{a_{1}+b_{1}}-\left\{\Gamma(2-\beta)\left[\int_{0}^{1} \frac{(1-\tau)^{\alpha-\beta-1}}{\Gamma(\alpha-\beta)} f(\tau, x(\tau)) d \tau-\frac{c_{2}}{b_{2}}\right]\right. \\
\left.-\frac{\Gamma(3-\gamma)}{2-\beta}\left[\int_{0}^{1} \frac{(1-\tau)^{\alpha-\gamma-1}}{\Gamma(\alpha-\gamma)} f(\tau, x(\tau)) d \tau-\frac{c_{3}}{b_{3}}\right]\right\} t \\
-\left\{\frac{\Gamma(3-\gamma)}{2}\left[\int_{0}^{1} \frac{(1-\tau)^{\alpha-\gamma-1}}{\Gamma(\alpha-\gamma)} f(\tau, x(\tau)) d \tau-\frac{c_{3}}{b_{3}}\right]\right\} t^{2} .
\end{gathered}
$$

\section{Main Results}

The following conditions are crucial to prove our results:

$\left(H_{1}\right)$ : The function $f:[0,1] \times \mathbb{R} \rightarrow \mathbb{R}$ satisfies: 


$$
|f(t, u)-f(t, \bar{u})| \leq k(t)|u-\bar{u}| ; t \in[0,1], u, \bar{u} \in \mathbb{R},
$$

where $k$ a continuous function on $[0,1]$.

$\left(H_{2}\right)$ : The function $f$ is continuous on $[0,1] \times \mathbb{R}$.

$\left(H_{3}\right)$ : There exists a positive constant $N$ such that $|f(t, u)| \leq N$ for any $t \in[0,1]$ and $u \in \mathbb{R}$.

Our first result is given by:

Theorem 3.1 Suppose that $\left(H_{1}\right)$ holds. If

$$
\begin{aligned}
& \|k\|\left[\left(\frac{1}{\Gamma(\alpha+1)}+\frac{\Gamma(2-\beta)}{\Gamma(\alpha-\beta+1)}\right)\left(\frac{\left|b_{1}\right|}{\left|a_{1}+b_{1}\right|}+1\right)\right. \\
& \left.+\frac{(2-\beta)^{-1}}{\Gamma(\alpha-\gamma+1)}\left(\frac{\left|b_{1}\right| \beta}{\left|a_{1}+b_{1}\right|}+\frac{4-\beta}{2} \Gamma(3-\gamma)\right)\right]<1,
\end{aligned}
$$

then the problem $(1)$ has a unique solution in $C([0,1], \mathbb{R})$.

Proof: To prove this theorem, we need to prove that the operator $\Psi$ has a fixed point on $C([0,1], \mathbb{R})$.

Let $x, \bar{x} \in C([0,1], \mathbb{R})$. Then for any $t \in[0,1]$, we have

$$
\begin{gathered}
|\Psi x(t)-\Psi \bar{x}(t)| \leq \int_{0}^{t} \frac{(t-\tau)^{\alpha-1}}{\Gamma(\alpha)}|f(\tau, x(\tau))-f(\tau, \bar{x}(\tau))| d \tau \\
+\frac{\left|b_{1}\right|}{\left|a_{1}+b_{1}\right|} \int_{0}^{1} \frac{(1-\tau)^{\alpha-1}}{\Gamma(\alpha)}|f(\tau, x(\tau))-f(\tau, \bar{x}(\tau))| d \tau \\
+\Gamma(2-\beta)\left(\frac{\left|b_{1}\right|}{\left|a_{1}+b_{1}\right|}+1\right) \int_{0}^{1} \frac{(1-\tau)^{\alpha-\beta-1}}{\Gamma(\alpha-\beta)}|f(\tau, x(\tau))-f(\tau, \bar{x}(\tau))| d \tau \\
+\frac{\beta}{2-\beta} \frac{\left|b_{1}\right|}{\left|a_{1}+b_{1}\right|} \int_{0}^{1} \frac{(1-\tau)^{\alpha-\gamma-1}}{\Gamma(\alpha-\gamma)}|f(\tau, x(\tau))-f(\tau, \bar{x}(\tau))| d \tau \\
+\left(\frac{4-\beta}{4-2 \beta} \Gamma(3-\gamma)\right) \int_{0}^{1} \frac{(1-\tau)^{\alpha-\gamma-1}}{\Gamma(\alpha-\gamma)}|f(\tau, x(t))-f(t, \bar{x}(\tau))| d \tau .
\end{gathered}
$$

Thanks to $\left(H_{1}\right)$, we obtain 


$$
\begin{gathered}
\|\Psi x-\Psi \bar{x}\| \leq\|k\|\left[\left(\frac{1}{\Gamma(\alpha+1)}+\frac{\Gamma(2-\beta)}{\Gamma(\alpha-\beta+1)}\right)\left(\frac{\left|b_{1}\right|}{\left|a_{1}+b_{1}\right|}+1\right)\right. \\
\left.+\frac{(2-\beta)^{-1}}{\Gamma(\alpha-\gamma+1)}\left(\frac{\left|b_{1}\right| \beta}{\left|a_{1}+b_{1}\right|}+\frac{4-\beta}{2} \Gamma(3-\gamma)\right)\right]\|x-\bar{x}\| .
\end{gathered}
$$

Using the condition (8), we conclude that $\Psi$ is a contraction mapping. Hence, by Banach fixed point theorem, there exists a unique fixed point in $C([0,1], \mathbb{R})$, which is a solution of the problem (1).

Corollary 3.2 Suppose that $f$ is an L-lipschitzian function. If

$$
\begin{aligned}
& L\left[\left(\frac{1}{\Gamma(\alpha+1)}+\frac{\Gamma(2-\beta)}{\Gamma(\alpha-\beta+1)}\right)\left(\frac{\left|b_{1}\right|}{\left|a_{1}+b_{1}\right|}+1\right)\right. \\
+ & \left.\frac{(2-\beta)^{-1}}{\Gamma(\alpha-\gamma+1)}\left(\frac{\left|b_{1}\right| \beta}{\left|a_{1}+b_{1}\right|}+\frac{4-\beta}{2} \Gamma(3-\gamma)\right)\right]<1,
\end{aligned}
$$

then the problem (1) has a unique solution on $[0,1]$.

Now, we discuss the existence of solution using Schaefer fixed point theorem. We have:

Theorem 3.3 Suppose that $\left(H_{2}\right)$ and $\left(H_{3}\right)$ are satisfied. Then the problem (1) has at least one solution in $C([0,1], \mathbb{R})$. steps:

Proof: We prove that $\Psi$ has a fixed point on $C([0,1], \mathbb{R})$ in the following

Step1: $\Psi$ is continuous on $C([0,1], \mathbb{R})$ :

Let $\left(x_{n}\right)_{n}$ be a sequence such that $x_{n} \longrightarrow x$ in $C([0,1], \mathbb{R})$. For each $t \in[0,1]$, we calculate $\left|\Psi x_{n}(t)-\Psi x(t)\right|$. We have 


$$
\begin{gathered}
\left|\Psi x_{n}(t)-\Psi x(t)\right| \leq \int_{0}^{t} \frac{(t-\tau)^{\alpha-1}}{\Gamma(\alpha)}\left|f\left(\tau, x_{n}(\tau)\right)-f(\tau, x(\tau))\right| d \tau \\
+\frac{\left|b_{1}\right|}{\left|a_{1}+b_{1}\right|} \int_{0}^{1} \frac{(1-\tau)^{\alpha-1}}{\Gamma(\alpha)}\left|f\left(\tau, x_{n}(\tau)\right)-f(\tau, x(\tau))\right| d \tau \\
+\Gamma(2-\beta)\left(\frac{\left|b_{1}\right|}{\left|a_{1}+b_{1}\right|}+1\right) \int_{0}^{1} \frac{(1-\tau)^{\alpha-\beta-1}}{\Gamma(\alpha-\beta)}\left|f\left(\tau, x_{n}(\tau)\right)-f(\tau, x(\tau))\right| d \tau \\
+\frac{\beta}{2-\beta} \frac{\left|b_{1}\right|}{\left|a_{1}+b_{1}\right|} \int_{0}^{1} \frac{(1-\tau)^{\alpha-\gamma-1}}{\Gamma(\alpha-\gamma)}\left|f\left(\tau, x_{n}(\tau)\right)-f(\tau, x(\tau))\right| d \tau \\
+\left(\frac{4-\beta}{4-2 \beta} \Gamma(3-\gamma)\right) \int_{0}^{1} \frac{(1-\tau)^{\alpha-\gamma-1}}{\Gamma(\alpha-\gamma)}\left|f\left(\tau, x_{n}(t)\right)-f(\tau, x(\tau))\right| d \tau .
\end{gathered}
$$

By $\left(H_{2}\right)$, we obtain

$$
\left|\Psi x_{n}-\Psi x\right| \longrightarrow 0, n \longrightarrow \infty
$$

Step2: $\Psi$ maps bounded sets into bounded sets of $C([0,1], \mathbb{R})$ :

For any $v>0$, we consider $B_{v}=\{x \in C([0,1], \mathbb{R}),\|x\| \leq v\}$.

Using $\left(H_{3}\right)$, we can write:

$$
\begin{aligned}
&\|\Psi x\| \leq \frac{\left|c_{1}\right|}{\left|a_{1}+b_{1}\right|}+\left(\frac{\left|b_{1}\right|}{\left|a_{1}+b_{1}\right|}+1\right)\left(\frac{N}{\Gamma(\alpha+1)}\right) \\
&+\Gamma(2-\beta)\left(\frac{\left|b_{1}\right|}{\left|a_{1}+b_{1}\right|}+1\right)\left(\frac{N}{\Gamma(\alpha-\beta+1)}+\frac{\left|c_{2}\right|}{\left|b_{2}\right|}\right) \\
&+\frac{\beta}{2-\beta} \frac{\left|b_{1}\right|}{\left|a_{1}+b_{1}\right|}+\frac{4-\beta}{4-2 \beta} \Gamma(3-\gamma),
\end{aligned}
$$

and consequently,

$$
\|\Psi x\|<\infty
$$

Step3: $\Psi$ maps bounded sets into equi-continuous sets of $C([0,1], \mathbb{R})$ : Let $t_{1}, t_{2} \in[0,1] ; t_{1}<t_{2}$ and let $x \in C([0,1], \mathbb{R})$. Then, we have 


$$
\begin{gathered}
\left|\Psi x\left(t_{2}\right)-\Psi x\left(t_{1}\right)\right| \leq\left(t_{2}-t_{1}\right)^{\alpha} \frac{2 N}{\Gamma(\alpha+1)}+\left(t_{2}^{\alpha}-t_{1}^{\alpha}\right) \frac{N}{\Gamma(\alpha+1)} \\
+\left(t_{2}^{2}-t_{1}^{2}\right)\left(\frac{\Gamma(3-\gamma)}{2}\left[\int_{0}^{1} \frac{(1-\tau)^{\alpha-\gamma-1}}{\Gamma(\alpha-\gamma)} f(t, x(t)) d \tau-\frac{c_{3}}{b_{3}}\right]\right) \\
+\left(t_{2}-t_{1}\right)\left(\Gamma(2-\beta)\left[\int_{0}^{1} \frac{(1-\tau)^{\alpha-\beta-1}}{\Gamma(\alpha-\beta)} f(t, x(t)) d \tau-\frac{c_{2}}{b_{2}}\right]\right. \\
\left.-\frac{\Gamma(3-\gamma)}{(2-\beta)}\left[\int_{0}^{1} \frac{(1-\tau)^{\alpha-\gamma-1}}{\Gamma(\alpha-\gamma)} f(t, x(t)) d \tau-\frac{c_{3}}{b_{3}}\right]\right) .
\end{gathered}
$$

As $t_{1} \rightarrow t_{2}$, the right-hand side of the above inequality tends to zero. Then, as a consequence of Steps 1, 2, 3 together with the Arzela-Ascoli theorem, we can conclude that $\Psi$ is completely continuous.

Step4: Now, we prove the boundeness of the set

$$
\Omega=\{x \in C([0,1], \mathbb{R}), x=\lambda \Psi x, 0<\lambda<1\} .
$$

Let $x \in \Omega$, then $x=\lambda \Psi x$ for some $0<\lambda<1$. Using (14), we get:

$$
\|x\|=\lambda\|\Psi x\|
$$

Therefore,

$$
\begin{aligned}
&\|x\| \leq \lambda\left[\frac{\left|c_{1}\right|}{\left|a_{1}+b_{1}\right|}+\left(\frac{\left|b_{1}\right|}{\left|a_{1}+b_{1}\right|}+1\right)\left(\frac{N}{\Gamma(\alpha+1)}\right)\right] \\
&+\lambda \Gamma(2-\beta)\left(\frac{\left|b_{1}\right|}{\left|a_{1}+b_{1}\right|}+1\right)\left(\frac{N}{\Gamma(\alpha-\beta+1)}+\frac{\left|c_{2}\right|}{\left|b_{2}\right|}\right) \\
&+\frac{\lambda \beta}{2-\beta} \frac{\left|b_{1}\right|}{\left|a_{1}+b_{1}\right|}+\frac{\lambda(4-\beta)}{4-2 \beta} \Gamma(3-\gamma) .
\end{aligned}
$$

Thus,

$$
\|x\|<\infty
$$

Finally, by Schaefer fixed point theorem, we deduce that $\Phi$ has a fixed point, which is a solution of the problem (1). 


\section{Examples}

Example 4.1 Consider the problem:

$$
\begin{gathered}
D^{\frac{5}{2}} x(t)=\frac{1}{t^{4}+3}\left(\frac{\sin x}{25}+\ln \left(t^{2}+1\right)\right), \quad t \in[0,1], \\
3 x(0)+x(1)=\frac{3}{4} \\
\frac{1}{2} D^{\frac{1}{2}} x(0)+\frac{5}{2} D^{\frac{1}{2}} x(1)=2 \\
4 D^{\frac{3}{2}} x(0)+\frac{3}{2} D^{\frac{3}{2}} x(1)=\frac{4}{5} .
\end{gathered}
$$

We have $\alpha=\frac{5}{2}, \beta=\frac{1}{2}, \gamma=\frac{3}{2}, a_{1}=3, a_{2}=\frac{1}{2}, a_{3}=4, b_{1}=1, b_{2}=\frac{5}{2}$, $b_{3}=\frac{3}{2}, c_{1}=\frac{3}{4}, c_{2}=2, c_{3}=\frac{4}{5}, f(t, x)=\frac{1}{t^{4}+3}\left(\frac{\sin x}{25}+\ln \left(t^{2}+1\right)\right)$.

We have also

$$
|f(t, x)-f(t, y)| \leq \frac{1}{75}|x-y|, t \in[0,1], x, y \in \mathbb{R}
$$

and

$$
\begin{aligned}
& \|k\|\left[\left(\frac{1}{\Gamma(\alpha+1)}+\frac{\Gamma(2-\beta)}{\Gamma(\alpha-\beta+1)}\right)\left(\frac{\left|b_{1}\right|}{\left|a_{1}+b_{1}\right|}+1\right)\right. \\
& \left.+\frac{(2-\beta)^{-1}}{\Gamma(\alpha-\gamma+1)}\left(\frac{\left|b_{1}\right| \beta}{\left|a_{1}+b_{1}\right|}+\frac{4-\beta}{2} \Gamma(3-\gamma)\right)\right] \\
& \approx \frac{1}{75}(0,925+1,112)=0,02713<1 .
\end{aligned}
$$

Thus, by Theorem 3.1, the problem (19) has a unique solution on [0,1].

Example 4.2 Consider the following boundary value problem

$$
\begin{gathered}
D^{\frac{7}{3}} x(t)=\frac{\cos (t x(t))}{e^{t}+1}, \quad t \in[0,1], \\
x(0)+2 x(1)=0, \\
3 D^{\frac{4}{5}} x(0)+\frac{1}{2} D^{\frac{4}{5}} x(1)=1, \\
-D^{\frac{4}{3}} x(0)+\frac{5}{2} D^{\frac{4}{3}} x(1)=\frac{4}{7} .
\end{gathered}
$$

It is clear that: $\alpha=\frac{7}{3}, \beta=\frac{4}{5}, \gamma=\frac{4}{3}, a_{1}=1, a_{2}=3, a_{3}=-1, b_{1}=2$, $b_{2}=\frac{1}{2}, b_{3}=\frac{5}{2}, c_{1}=0, c_{2}=1, c_{3}=\frac{4}{7}$, and $f(t, x)=\frac{\cos (t x)}{e^{t}+1}$. 
The hypotheses $\left(H_{1}\right)$ and $\left(H_{2}\right)$ are satisfied. Consequently, by Theorem 3.3, the problem (20) has at least a solution on $[0,1]$.

\section{Open Problems}

We propose the following open problems:

Open Problem 1: Using Caputo approach for fractional differential operator of order $\alpha$, under what conditions do Theorems 3.1 and Theorem 3.3 hold for $n<\alpha<n+1, n \in \mathbb{N}^{*}$ ?

Open Problem 2: Is it possible to generalize the above main results for (1), where the fractional derivatives are taken in the sense of Riemann-Liouville and $n<\alpha<n+1, n \in \mathbb{N}$ ?

\section{References}

[1] K. Assaleh, W.M. Ahmed: Modeling of speech signals using fractional calculus . 9th International Symposium on Signal Processing and Its Applications. 12 (15), (2007), pp.1-4.

[2] M.A. Bengrine, Z. Dahmani: Boundary vale problems for fractional differential equations. International Journal of Open Problems Computer Science and Mathematics. 3 (1), (2012), pp. 2074-2827.

[3] K. Diethelm, N.J. Ford: Analysis of fractional differential equations. Journal of Mathematics Analysis and Applications. 265 (2), (2002), pp. 229248.

[4] A.L. Gabriele, D. Merlini, T.F. Nonnenmacher, E.R. Weibel: Fractals in biology and medecine. Birkhauser Verlag, (2005).

[5] A. Kaur, P.S. Takhar, D.M. Smith, J.E. Mann, M.M. Brashears: Fractional differential equations based modeling of microbial survival and growth curves: Model development and experimental validation. Journal of Food Science. 73 (8), (2008), pp. 403-414.

[6] A.A. Kilbas, H.M. Srivastava, J.J. Trujillo: The theory and application of fractional differential equation. North-Holand Mathematics Studies. Elsevier, (2006).

[7] V.V. Kulish, J. L. Lage: Application of fractional calculus to fluid mecanics. Journal of Fluids Engineering. 124 (3), (2002), pp. 803-807. 
[8] V. Lakshmikantham, A.S. Vatsala: Basic theory of fractional differential equations. Nonlinear Analysis Theory, Methods. 69 (8), (2008), pp. 26772682 .

[9] X. Liu, Y. Liu: Fractional differential equations with fractional nonseparated boundary conditions. Electronic Journal of Diffential Equations. 25, (2013), pp. 1-13.

[10] R.L. Magin: Fractional calculus in bioengineering. Critical Reviews in Biomedical Engineering. 32 (1), (2004), pp. 1-104.

[11] T.S. Margulies: Wave propagation in viscoelastic horns a fractional calculus rheology model. Acoustical Society of Americ Journal. 114 (4), (2003), pp. 2424-2442.

[12] B. Mathieu, P. Melchior, A. Oustaloup, Ch. Ceyral: Fractional differentiation for edge detection. Fractional Signal Processiong and Applications. 83 (11), (2003), pp. 2285-2480.

[13] K.S. Miler, B. Ross: An introdution to the fractional calculus and fractional differential equations. J. Wiley, New York, (1993).

[14] K.B. Oldham, J. Spanier: The fractional calculus. Academic Press, (1974).

[15] I. Podlubny: Fractional differential equations. Mathematics in science and Engineering. Academic press, New York (1998).

[16] I. Podlubny, I. Petras, B.M. Vinagre, P.O. Leray, L. Dorcak: Analogue realizations of fractional order controllers. Nonlinear Dynamics. 29 (1-4), (2002), pp. 281-296.

[17] J. Sabatier, O.P. agrawal, J.A.T. Manchado: Advances in fractional calculus: Theorical developments and applications in physics andengineering. Dordrecht, Springer, (2007).

[18] S.G. Samko, A.A. Kilbas, O.I. Marichev: Fractional integrals andderivatives: Theory and applications. Yverdon,Gordon and Breach, (1993).

[19] E. Soczkiewicz: Application of fractional calculus in the theory of viscoelasticity. Molecular and Quantum Acoustics. 23, (2002), pp. 397-404.

[20] J.I. Suarez, B.M. Vinagre, A.J. Calderon, C.A. Monje, Y.Q. Chen: Using fractional calculus for lateral and longitudinal controle of autonomous vehicles. Lecture Notes in Computer Science. Springer (2809), (2004). 
[21] S. Zhang: Positive solutions for boundary-value problems of nonlinear fractionaldiffrential equations. Electronic Journal of Differential Equation. 36 (1-12), (2006), pp. 37-44. 